\title{
Prognostic predictive values of gemcitabine sensitivity-related gene products for unresectable or recurrent biliary tract cancer treated with gemcitabine alone
}

\author{
Akihiro Murata, Ryosuke Amano ${ }^{*}$, Nobuya Yamada, Kenjiro Kimura, Masakazu Yashiro, Bunzo Nakata
} and Kosei Hirakawa

\begin{abstract}
Background: Gemcitabine is a pyrimidine nucleoside analog that is a commonly used chemotherapeutic agent for unresectable or recurrent biliary tract cancer (BTC). Several molecules involved in gemcitabine metabolism, including human equilibrative nucleoside transporter (hENT1), deoxycytidine kinase (dCK), and ribonucleotide reductase subunit M1 (RRM1), have been investigated as predictive biomarkers of gemcitabine efficacy, mostly in pancreatic cancer. The aim of this study is to clarify which biomarker is the most reliable among hENT1, dCK, and RRM1 to predict survival in patients with advanced BTC treated with gemcitabine alone.

Methods: The analysis was performed on samples from 28 patients with unresectable or recurrent BTC who were treated with gemcitabine alone as first-line therapy. The starting date of overall survival (OS) and progression-free survival (PFS) was defined as the date of first treatment with gemcitabine. Intratumoral hENT1, dCK, and RRM1 expressions were examined by immunohistochemistry.
\end{abstract}

Results: The expressions of hENT1, dCK, and RRM1 had no significant relationships with age, gender, primary tumor site, recurrence/unresectable, or histological type. Among the three molecules, only hENT1 expression was a significant factor affecting OS and PFS in univariate analysis; OS was 11.4 months for high hENT1 expression versus 5.7 months for low, $P=0.0057$; PFS was 7.7 months for high versus 2.5 months for low, $P=0.0065$. Multivariate analyses also identified hENT1 expression as an independent predictive factor for OS.

Conclusions: hENT1 is the most reliable predictive marker of survival in patients with advanced BTC treated with gemcitabine.

Keywords: Biliary tract cancer, Deoxycytidine kinase, Gemcitabine, Human equilibrative nucleoside transporter 1, Ribonucleotide reductase subunit M1

\section{Background}

Biliary tract cancer (BTC) is relatively rare, but its incidence is increasing worldwide. The prevalence is much higher in East Asia and Latin America than in Europe and the United States [1,2]. Although a complete surgical resection is the only curative modality, most patients are not eligible for surgery because of the advanced stage of disease at diagnosis. Moreover, even patients who undergo

\footnotetext{
* Correspondence: ramano@med.osaka-cu.ac.jp

Department of Surgical Oncology, Osaka City University Graduate School of Medicine, 1-4-3 Asahi-machi, Abeno-ku, Osaka 545-8585, Japan
}

a surgical resection often have a recurrence of the disease. The outcome for patients with unresectable or recurrent BTC is dismal and their median survival is usually under 1 year [3]. Most patients with unresectable or recurrent BTC are therefore candidates for palliative chemotherapy.

Gemcitabine $\left(2^{\prime}, 2^{\prime}\right.$-difluorodeoxycytidine), a deoxycytidine analog that inhibits DNA replication and repair, is the most effective single agent in advanced BTC. Because gemcitabine is hydrophilic and does not cross the plasma membrane by passive diffusion, its cellular uptake requires the presence of a specialized plasma membrane nucleoside

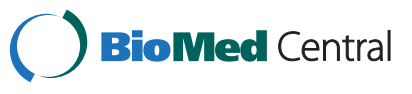


transporter [4]. Gemcitabine is transported into the cell mainly by human equilibrative transporter 1 (hENT1). After intracellular entry, gemcitabine is phosphorylated by deoxycytidine kinase $(\mathrm{dCK})$ to its active diphosphate and triphosphate in a rate-limiting step. The incorporation of gemcitabine triphosphate into DNA, leading to chain termination, is a major mechanism underlying the cytotoxicity of gemcitabine [5,6]. In addition, gemcitabine diphosphate inhibits ribonucleotide reductase (RRM1, RRM2), causing a decrease in the cellular pool of deoxycytidine triphosphate that competes with gemcitabine triphosphate for incorporation into DNA [7].

Recent investigations using cell lines or surgical specimens have revealed that these proteins were predictors for the efficacy of gemcitabine treatment. In particular, hENT1 expression has been evaluated as a predictive marker for gemcitabine chemotherapy in patients with pancreatic cancer. In vitro studies demonstrated that deficiency of hENT1 conferred resistance to gemcitabine and hENT1 expression was positively associated with gemcitabine chemosensitivity $[4,8,9]$. In patients with pancreatic cancer from a randomized phase III RTOG 9704 study, hENT1 expression was associated with increased overall survival (OS) and diseasefree survival in patients who received adjuvant gemcitabine chemotherapy, but not in those who received 5-fluorouracil [10]. Other retrospective studies have also demonstrated the predictive and prognostic value of hENT1 in patients with pancreatic cancer $[11,12]$.

Other key enzymes involved in gemcitabine metabolism have also been evaluated as predictive markers. In pancreatic cancer, high dCK expression was identified as an independent prognostic factor in patients who received adjuvant gemcitabine therapy [13]. In contrast, high expression of RRM1 was associated with poor survival after gemcitabine treatment in patients with recurrent pancreatic cancer [14,15].

There are thus many reports about predictive markers for the efficacy of gemcitabine in pancreatic cancer, but there are limited data available on the predictive value of these markers in BTC. Based on the importance of the biomarkers involved in gemcitabine metabolism, we assessed the expressions of three key molecules (hENT1, dCK, and RRM1) in tumor samples from 28 patients with advanced BTC who received first-line gemcitabine monotherapy. To our knowledge, this study is the first to examine the predictive aspect of hENT1, dCK, and RRM1 for gemcitabinetreated advanced BTC in the same clinical samples. The aim of this study was to investigate the association between the expressions of these proteins and prognosis.

\section{Methods}

\section{Subjects}

A total of 28 patients with histopathologically confirmed unresectable or postoperative recurrent BTC treated
Table 1 Subject characteristics

\begin{tabular}{ll}
\hline Total number & $\mathbf{2 8}$ \\
\hline Age (years) & 64 \\
Median & 46 to 81 \\
Range & \\
Gender & 15 \\
Male & 13 \\
Female & \\
Primary tumor site & 18 \\
Extrahepatic bile duct & 10 \\
Gallbladder & \\
Recurrence or unresectable & 22 \\
Recurrence after surgery & 6 \\
Unresectable &
\end{tabular}

with first-line gemcitabine monotherapy at Osaka City University Hospital between October 2006 and April 2011 were included in this study. Adjuvant chemotherapy including gemcitabine was not given to these patients. BTC comprised extrahepatic bile duct cancer and gallbladder cancer. Intrahepatic bile duct cancer was excluded because unresectable or recurrent intrahepatic bile duct cancer patients in our institution were treated

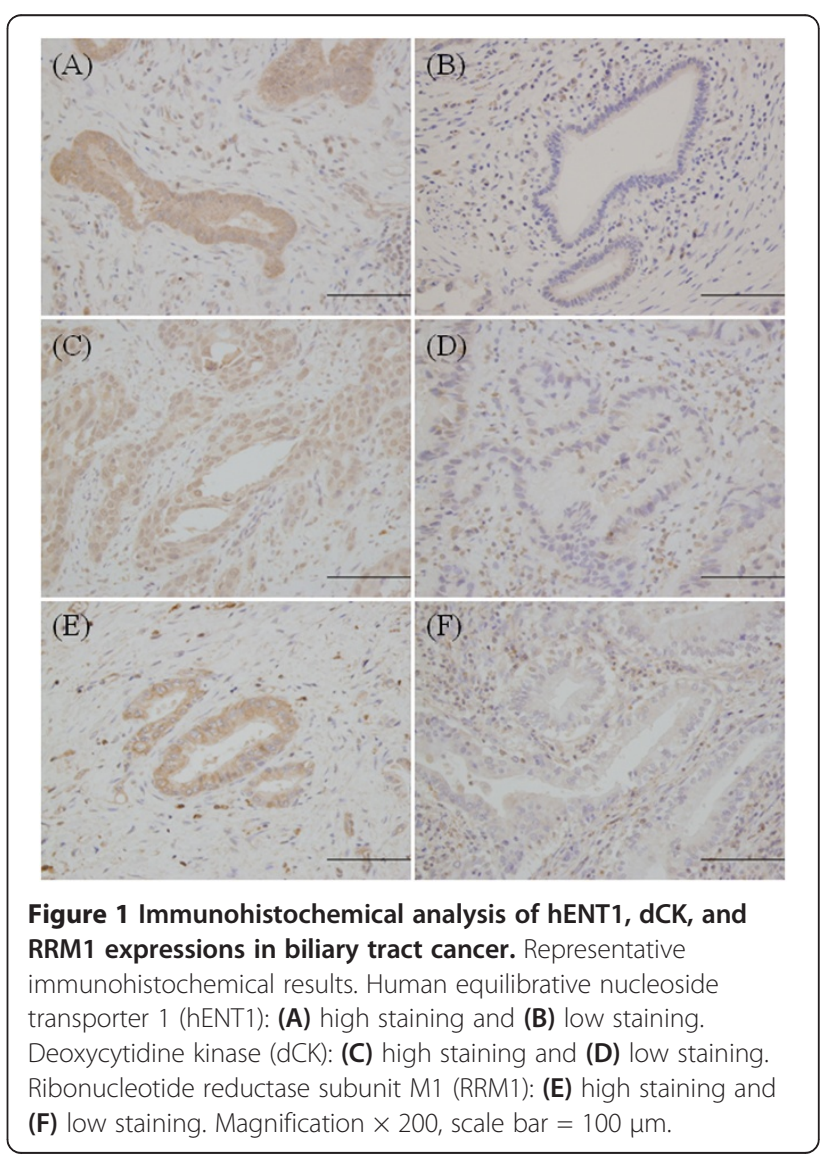


Table 2 Clinicopathological characteristics based on hENT1, dCK, and RRM1 expression for advanced biliary tract cancer subjects

\begin{tabular}{|c|c|c|c|c|c|c|c|c|c|}
\hline \multirow[t]{2}{*}{ Characteristic } & \multicolumn{2}{|c|}{ hENT1 expression } & \multirow[t]{2}{*}{$P$ value } & \multicolumn{2}{|c|}{ dCK expression } & \multirow[t]{2}{*}{$P$ value } & \multicolumn{2}{|c|}{ RRM1 expression } & \multirow[t]{2}{*}{$P$ value } \\
\hline & $\begin{array}{c}\text { Low } \\
(n=11)\end{array}$ & $\begin{array}{c}\text { High } \\
(n=17)\end{array}$ & & $\begin{array}{c}\text { Low } \\
(n=13)\end{array}$ & $\begin{array}{c}\text { High } \\
(n=15)\end{array}$ & & $\begin{array}{c}\text { Low } \\
(n=11)\end{array}$ & $\begin{array}{c}\text { High } \\
(n=17)\end{array}$ & \\
\hline \multicolumn{10}{|l|}{ Primary tumor site } \\
\hline Extrahepatic bile duct & 7 & 11 & 1.000 & 10 & 8 & 0.254 & 8 & 10 & 0.689 \\
\hline Gallbladder & 4 & 6 & & 3 & 7 & & 3 & 7 & \\
\hline \multicolumn{10}{|l|}{ Recurrence or unresectable } \\
\hline Recurrence after surgery & 9 & 13 & 1.000 & 9 & 13 & 0.372 & 7 & 15 & 0.174 \\
\hline Unresectable & 2 & 4 & & 4 & 2 & & 4 & 2 & \\
\hline \multicolumn{10}{|l|}{ Histological type } \\
\hline Well/moderately & 9 & 12 & 0.668 & 11 & 10 & 0.396 & 9 & 12 & 0.668 \\
\hline Poor & 2 & 5 & & 2 & 5 & & 2 & 5 & \\
\hline \multicolumn{10}{|l|}{ hENT1 expression } \\
\hline High & - & - & - & 7 & 10 & 0.700 & 6 & 11 & 0.701 \\
\hline Low & - & - & & 6 & 5 & & 5 & 6 & \\
\hline \multicolumn{10}{|l|}{ dCK expression } \\
\hline High & * & * & * & - & - & - & 2 & 13 & 0.006 \\
\hline Low & * & * & & - & - & & 9 & 4 & \\
\hline
\end{tabular}

dCK, deoxycytidine kinase; hENT1, human equilibrative nucleoside transporter; RRM1, ribonucleotide reductase subunit M1. *Same as above line (hENT1 expression).

mostly with systemic gemcitabine plus hepatic arterial infusion of 5-fluorouracil. Subject demographics and clinical characteristics are listed in Table 1. In six subjects with unresectable cases, one had liver metastasis and five had peritoneal disseminations. The median OS from initiation of gemcitabine chemotherapy was 10.0 months for all 28 subjects. All tumor samples were obtained prior

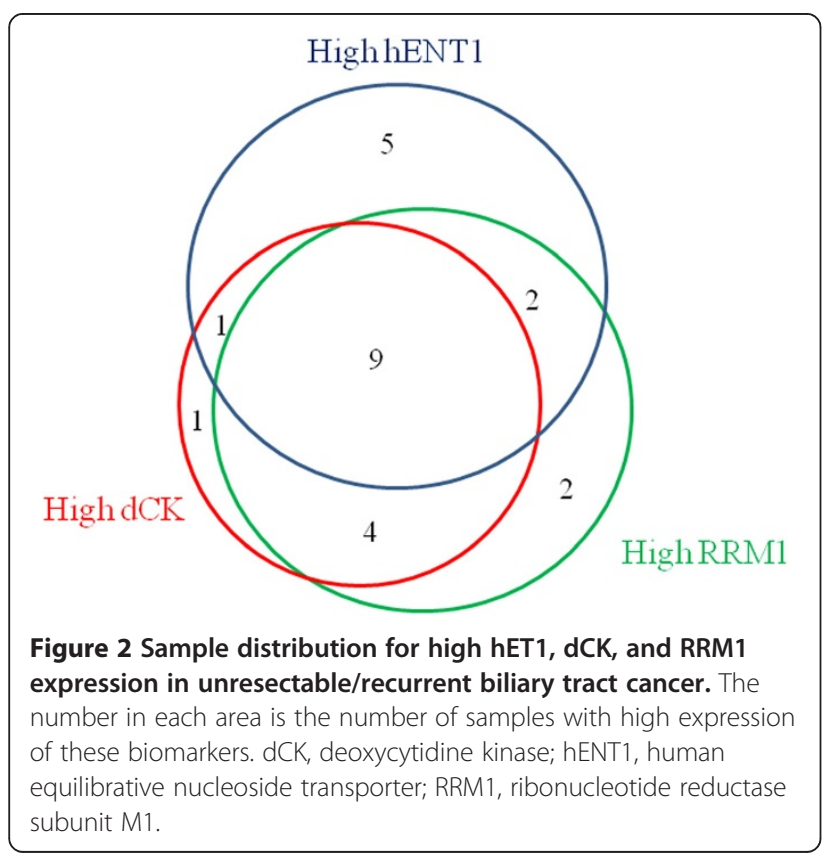

to gemcitabine chemotherapy. For all six unresectable tumors, the biopsy specimens were obtained from metastatic lesions during probe laparotomies. Chemotherapy consisted of intravenous gemcitabine infusion using the following protocols: the gemcitabine standard protocol $\left(1,000 \mathrm{mg} / \mathrm{m}^{2}\right.$ on days 1,8 , and 15 every 4 weeks). When the patients treated with weekly gemcitabine presented a grade 3 hematological adverse event or a grade 2 nonhematological adverse event defined by Common Terminology Criteria for Adverse Events version 3.0, a biweekly protocol (1,000 $\mathrm{mg} / \mathrm{m}^{2}$ on days 1 and 15 every 4 weeks) was given to them. Informed consent to use the specimens for this study according to the institutional rules of the hospital was obtained from all subjects.

\section{Immunohistochemistry of the specimens}

Biomarker (hENT1, dCK, and RRM1) expression in BTC specimens was determined by immunohistochemical staining using the avidin-biotin-peroxidase complex method. In brief, the $4 \mu \mathrm{m}$ thick formalin-fixed, paraffinembedded sections were deparaffinized in xylene and decreasing concentrations of ethanol. The slides were treated with 3\% hydrogen peroxide in methanol for 15 minutes to block endogenous peroxidase activity. To retrieve the antigenicities, slides were heated for 10 minutes at $105^{\circ} \mathrm{C}$ by autoclave in Target Retrieval Solution (Dako Co., Carpinteria, CA, USA) and cooled at room temperature for at least 30 minutes. Incubation was performed in a humidified chamber overnight at $4^{\circ} \mathrm{C}$ with anti- 
Table 3 Univariate analysis for overall survival by logrank test

\begin{tabular}{|c|c|c|c|}
\hline Variable & Number & $\begin{array}{c}\text { Median overall } \\
\text { survival (months) }\end{array}$ & $P$ value \\
\hline \multicolumn{4}{|l|}{ Primary tumor site } \\
\hline Extrahepatic bile duct & 18 & 11.4 & 0.2423 \\
\hline Gallbladder & 10 & 9.2 & \\
\hline \multicolumn{4}{|l|}{ Recurrence or unresectable } \\
\hline Recurrence & 22 & 10.0 & 0.6183 \\
\hline Unresectable & 6 & 9.2 & \\
\hline \multicolumn{4}{|l|}{ Histological type } \\
\hline Well/moderately & 21 & 10.0 & 0.9202 \\
\hline Poor & 7 & 11.4 & \\
\hline \multicolumn{4}{|l|}{ hENT1 expression } \\
\hline Low & 11 & 5.7 & 0.0057 \\
\hline High & 17 & 11.4 & \\
\hline \multicolumn{4}{|l|}{ dCK expression } \\
\hline Low & 13 & 9.2 & 0.3725 \\
\hline High & 15 & 11.4 & \\
\hline \multicolumn{4}{|l|}{ RRM1 expression } \\
\hline Low & 11 & 10.2 & 0.5941 \\
\hline High & 17 & 10.0 & \\
\hline
\end{tabular}

dCK, deoxycytidine kinase; hENT1, human equilibrative nucleoside transporter; RRM1, ribonucleotide reductase subunit M1.

hENT1 rabbit polyclonal antibody (Proteintech, Chicago, IL, USA) at a 1:100 dilution and with anti-RRM1 mouse $\mathrm{mAb}$ (Proteintech) at a 1:100 dilution. The final incubation was for 2 hours at room temperature with anti-dCK rabbit polyclonal antibody (LSBio, Seattle, WA, USA) at a 1:24 dilution $(20 \mu \mathrm{g} / \mathrm{ml}) ; 3,3^{\prime}$-diaminobenzidine (Nichirei, Tokyo, Japan) was used as a chromogen. The sections were rinsed, counterstained with hematoxylin, dehydrated through graded alcohol and xylene, and coverslipped. Negative controls processed by omitting the primary antibodies were included for each staining.

\section{Immunohistochemical evaluation}

As previously reported [10], the scoring for hENT1 was based on relative intensities of the BTC staining with reference to the normally strong hENT1 staining within lymphocytes. These internal references were used as internal positive controls between slides and samples, as well as for the staining procedure. Tumor tissue staining was then evaluated by comparison with the internal controls. A score of high hENT1 staining was given for weak and/or strong reactivity in $>50 \%$ of neoplastic cells. A score of low hENT1 staining was given if there was no staining in $>50 \%$ of cells. Scoring for dCK and RRM1 was done in the same manner on the basis of the relative intensities of tumor staining with reference to the internal controls. The internal control for dCK was provided by lymphocyte staining as previously described [13]. Plasma and stromal cells showed positive RRM1 staining for cytoplasm, which was considered the internal control [16]. Staining grade was evaluated by two investigators without previous knowledge of the clinical characteristics and outcomes.

\section{Statistical analysis}

All subjects were classified into high and low expression groups according to hENT1, dCK, and RRM1 staining. The significance of the correlation between expressions and clinicopathological characteristics was assessed by the chi-square test (Fisher's exact test). Survival probabilities were calculated using the Kaplan-Meier method, and the log-rank test was used for univariate survival analysis. If OS was defined as the period from the date of first diagnosis of BTC, various factors such as operative curability and resectability might affect the survival time. The purpose of this study was focused on the impact of gemcitabine sensitivity-related gene products. OS was therefore measured from the date of first treatment with gemcitabine to the date of death or last follow-up evaluation. Progressionfree survival (PFS) according to clinical judgment was measured from the date of first treatment with gemcitabine to the date of first progression or death without any progressive disease. Data on survivors were censored at the last follow-up. We also performed a multivariate analysis using Cox proportional hazards modeling to measure correlations between clinicopathological variables and OS. For all tests, two-sided $P$ values $<0.05$ were defined as statistically significant. The SPSS software program (SPSS Japan, Tokyo, Japan) was used for the analysis.

Table 4 Multivariate analysis for overall survival using variables including hENT1 and dCK

\begin{tabular}{|c|c|c|c|c|}
\hline Variable & Comparison & HR & $95 \% \mathrm{Cl}$ & $P$ value \\
\hline Primary tumor site & Gallbladder vs. extrahepatic bile duct & 1.617 & 0.596 to 4.389 & 0.345 \\
\hline Recurrence or unresectable & Unresectable vs. recurrence & 1.707 & 0.511 to 5.700 & 0.384 \\
\hline Histological type & Poor vs. well/moderately & 1.117 & 0.354 to 3.519 & 0.851 \\
\hline hENT1 expression & High vs. low & 0.270 & 0.097 to 0.748 & 0.012 \\
\hline dCK expression & High vs. low & 0.778 & 0.284 to 2.132 & 0.625 \\
\hline
\end{tabular}

$\mathrm{Cl}$, confidence interval; dCK, deoxycytidine kinase; hENT1, human equilibrative nucleoside transporter; HR, hazard ratio. 
Table 5 Multivariate analysis for overall survival using variables including hENT1 and RRM1

\begin{tabular}{|c|c|c|c|c|}
\hline Variable & Comparison & HR & $95 \% \mathrm{Cl}$ & $P$ value \\
\hline Primary tumor site & Gallbladder vs. extrahepatic bile duct & 2.136 & 0.751 to 6.078 & 0.155 \\
\hline Recurrence or unresectable & Unresectable vs. recurrence & 3.109 & 0.860 to 11.238 & 0.084 \\
\hline Histological type & Poor vs. well/moderately & 0.732 & 0.235 to 2.282 & 0.591 \\
\hline hENT1 expression & High vs. low & 0.220 & 0.077 to 0.629 & 0.005 \\
\hline RRM1 expression & High vs. low & 2.315 & 0.773 to 6.928 & 0.133 \\
\hline
\end{tabular}

$\mathrm{Cl}$, confidence interval; hENT1, human equilibrative nucleoside transporter; HR, hazard ratio; RRM1, ribonucleotide reductase subunit M1.

\section{Results}

\section{Immunostaining and subject background}

Examples of high and low tumor staining are shown in Figure 1. hENT1 immunostaining was localized predominantly in the membrane, although occasional cytoplasmic staining was also observed. dCK immunostaining was located in the cytoplasm and the nucleus and RRM1 staining was seen in the cytoplasm. Of the 28 tumors, hENT1 was high in 17 (60.7\%), dCK in 15 (53.6\%), and RRM1 in 17 (60.7\%). The relationship between clinicopathological factors and the expressions of hENT1, dCK, and RRM1 is summarized in Table 2. There were no significant differences in primary tumor site, recurrence/unresectable, or histological type. Among hENT1, dCK, and RRM1 expressions, a significant and strong association $(P=0.006)$ was

Table 6 Univariate analysis for progression free survival by log-rank test

\begin{tabular}{|c|c|c|c|}
\hline Variable & Number & $\begin{array}{l}\text { Progression-free } \\
\text { survival (months) }\end{array}$ & $P$ value \\
\hline \multicolumn{4}{|l|}{ Primary tumor site } \\
\hline Extrahepatic bile duct & 18 & 4.6 & 0.2551 \\
\hline Gallbladder & 10 & 3.4 & \\
\hline \multicolumn{4}{|l|}{ Recurrence or unresectable } \\
\hline Recurrence & 22 & 3.9 & 0.7073 \\
\hline Unresectable & 6 & 7.4 & \\
\hline \multicolumn{4}{|l|}{ Histological type } \\
\hline Well/moderately & 21 & 4.4 & 0.7255 \\
\hline Poor & 7 & 5.2 & \\
\hline \multicolumn{4}{|l|}{ hENT1 expression } \\
\hline Low & 11 & 2.5 & 0.0065 \\
\hline High & 17 & 7.7 & \\
\hline \multicolumn{4}{|l|}{ dCK expression } \\
\hline Low & 13 & 4.6 & 0.8076 \\
\hline High & 15 & 3.7 & \\
\hline \multicolumn{4}{|l|}{ RRM1 expression } \\
\hline Low & 11 & 7.4 & 0.1349 \\
\hline High & 17 & 2.5 & \\
\hline
\end{tabular}

dCK, deoxycytidine kinase; hENT1, human equilibrative nucleoside transporter; RRM1, ribonucleotide reductase subunit M1. observed between dCK and RRM1. Figure 2 shows the distribution of high expression of the three biomarkers.

\section{Survival analysis according to hENT1, dCK, and RRM1 expression}

Univariate analysis showed that the expression of hENT1 was associated significantly with OS; however, primary tumor site, recurrence/unresectable, histological type, dCK, and RRM1 were not associated with OS (Table 3). Confounding factors (dCK and RRM1) could not be analyzed simultaneously in a multivariate analysis. Therefore, each of dCK and RRM1 was added to the multivariate analysis with primary tumor site, recurrence or unresectable, histological type, and hENT1 expression. The results demonstrated that high hENT1 expression alone was an independent prognostic predictor (Tables 4 and 5). Univariate analysis for PFS demonstrated significant association between hENT1 and PFS. Other variables including $\mathrm{dCK}$ and RRM1 were not related to PFS (Table 6).

\section{Discussion}

hENT1 is the primary gatekeeper for intracellular uptake of gemcitabine, and both dCK and RRM1 are related to gemcitabine metabolism after intracellular entry. Marechal and colleagues recently investigated the predictive value of hENT1, dCK, and RRM1 in patients with pancreatic cancer treated with adjuvant gemcitabine chemotherapy and demonstrated that both hENT1 and dCK expressions were powerful predictive markers [17]. There have been several studies on the impact of single gemcitabine sensitivity-related gene products (such as hENT1, dCK, and RRM1) on gemcitabine-based treatment effects in BTC. These previous investigations were based on immunohistochemistry. To our knowledge, there have been no studies on the association between gemcitabine effects and mRNA expression of gemcitabine sensitivity-related genes in BTC. Our study was performed using the immunohistochemical method because the frozen tissues of BTC studied here were not kept. The originality of our study is that we observed all of these biomarkers simultaneously in patients with BTC treated only with gemcitabine.

We analyzed hENT1, dCK, and RRM1 expression using immunohistochemical analysis to determine the prognostic value in patients with advanced BTC treated 
with gemcitabine. hENT1 was the only independent predictive marker for gemcitabine chemotherapy in advanced BTC. Patients with a high level of tumor hENT1 had a statistically significant longer OS and PFS than those with low expression. Our study also demonstrated that high hENT1 expression was an independent prognostic factor in patients with advanced BTC, treated with first-line gemcitabine monotherapy.

There are a few papers on the prognostic value of hENT1 in patients with BTC treated with gemcitabine. Santini and colleagues reported that an immunohistochemical evaluation of intratumoral hENT1 expression might be useful in predicting the clinical outcome of gemcitabine-based chemotherapies in 31 patients with advanced BTC (extrahepatic biliary tract, intrahepatic biliary tract, gall bladder, and ampulla). However, they were unable to find a statistically significant difference in OS [18]. Borbath and colleagues reported that high expression of hENT1 was independently associated with prolonged PFS and OS in 26 patients with locally advanced or metastatic extrahepatic and intrahepatic cholangiocarcinoma who were treated with gemcitabine [19]. Kobayashi and colleagues recently demonstrated that high hENT1 expression was associated with prolonged OS in resected extrahepatic and intrahepatic cholangiocarcinoma treated with gemcitabine-based adjuvant chemotherapy [20]. The primary sites of BTC in these studies and ours varied. However, the positive survival impact of hENT1 on BTC treated with gemcitabine-based chemotherapy in these studies was concordant. On the other hand, the potential prognostic value of hENT1 expression in patients with BTC who did not receive gemcitabine chemotherapy is unclear. Although we investigated the expression of hENT1 in 39 patients with advanced BTC who did not receive gemcitabine, no significant survival difference was observed between patients with high and low hENT1 (data not shown).

RRM1 have been reported previously as predictive markers for gemcitabine in pancreatic cancer, although there are few published studies evaluating their value in BTC. Nakamura and colleagues reported that high RRM1 expression had a positive association with poor prognosis in 10 patients with advanced BTC treated with gemcitabine-based chemotherapy with or without radiation [21]. In the current study, the expression of RRM1 was not associated with a prognosis. The differing results for RRM1 impact on the survival of patients with BTC between Nakamura and colleagues' study and ours might be due to the different treatment modalities.

An abundance of dCK is commonly known to be associated with gemcitabine sensitivity in pancreatic cancer. There has been no paper describing dCK expression in patients with BTC. In the current study, there was significant correlation between $\mathrm{dCK}$ and RRM1 expression.
The reason for this is unclear, but both proteins are key enzymes related to deoxycytidine metabolism.

The limitation of this study is that it was a retrospective evaluation. Prospective investigation including an adequate number of samples is needed to confirm the importance of gemcitabine sensitivity-related gene products in patients with advanced BTC treated with gemcitabine. In addition, the relations between gemcitabine effects in BTC and other gemcitabine sensitivity-related gene products such as RRM2, cytidine deaminase, human concentrative nucleoside transporter 1 and 3, 5' -nucleotidase, and deoxycytidylate deaminase are remained to be elucidated for further investigations.

\section{Conclusions}

In this study, high hENT1 expression was a reliable predictive marker of survival in patients with recurrent or unresectable BTC who received gemcitabine chemotherapy alone.

\section{Abbreviations \\ BTC: Biliary tract cancer; dCK: Deoxycytidine kinase; hENT1: Human equilibrative nucleoside transporter $1 ;$ mAb: Monoclonal antibody; OS: Overall survival; PFS: Progression-free survival; RRM1: Ribonucleotide reductase subunit $\mathrm{M} 1$}

\section{Competing interests}

The authors declare that they have no competing interests.

\section{Authors' contributions}

AM carried out almost studies and performed the manuscript. RA and NY supported with design and interpretation of this study. Statistical analysis was performed by AM and KK. MY and BN helped to draft the manuscript. Overall supervision of the manuscript was completed by $\mathrm{KH}$. All authors read and approved the final manuscript.

Received: 12 December 2012 Accepted: 12 May 2013

Published: 27 May 2013

\section{References}

1. Randi G, Malvezzi M, Levi F, Ferlay J, Negri E, Franceschi S, La Vecchia C: Epidemiology of biliary tract cancers: an update. Ann Oncol 2009, 20:146-159.

2. Matsuda T, Marugame T: International comparisons of cumulative risk of gallbladder cancer and other biliary tract cancer, from Cancer Incidence in Five Continents Vol. VIII. Jpn J Clin Oncol 2007, 37:74-75.

3. Eckel F, Schmid RM: Chemotherapy in advanced biliary tract carcinoma: a pooled analysis of clinical trials. Br J Cancer 2007, 96:896-902.

4. Mackey JR, Mani RS, Selner M, Mowles D, Young JD, Belt JA, Crawford CR, Cass CE: Functional nucleoside transporters are required for gemcitabine influx and manifestation of toxicity in cancer cell lines. Cancer Res 1998, 58:4349-4357.

5. Huang P, Plunkett W: Induction of apoptosis by gemcitabine. Semin Oncol 1995, 22:19-25.

6. van Haperen WWR, Veerman G, Vermorken JB, Peters GJ: 2',2'-Difluorodeoxycytidine (gemcitabine) incorporation into RNA and DNA of tumour cell lines. Biochem Pharmacol 1993, 46:762-766.

7. Plunkett W, Huang P, Searcy CE, Gandhi V: Gemcitabine: preclinical pharmacology and mechanisms of action. Semin Oncol 1996, 23:3-15.

8. Nakano Y, Tanno S, Koizumi K, Nishikawa T, Nakamura K, Minoguchi M, Izawa T, Mizukami Y, Okumura T, Kohgo Y: Gemcitabine chemoresistance and molecular markers associated with gemcitabine transport and metabolism in human pancreatic cancer cells. Br J Cancer 2007, 96:457-463 
9. Mori R, Ishikawa T, Ichikawa Y, Taniguchi K, Matsuyama R, Ueda M, Fujii Y, Endo I, Togo S, Danenberg PV, Shimada H: Human equilibrative nucleoside transporter 1 is associated with the chemosensitivity of gemcitabine in human pancreatic adenocarcinoma and biliary tract carcinoma cells. Oncol Rep 2007, 17:1201-1205.

10. Farrell JJ, Elsaleh H, Garcia M, Lai R, Ammar A, Regine WF, Abrams R, Benson AB, Macdonald J, Cass CE, Dicker AP, Mackey JR: Human equilibrative nucleoside transporter 1 levels predict response to gemcitabine in patients with pancreatic cancer. Gastroenterology 2009, 136:187-195.

11. Spratlin J, Sangha R, Glubrecht D, Dabbagh L, Young JD, Dumontet C, Cass C, Lai R, Mackey JR: The absence of human equilibrative nucleoside transporter 1 is associated with reduced survival in patients with gemcitabine-treated pancreas adenocarcinoma. Clin Cancer Res 2004, 10:6956-6961.

12. Giovannetti E, Del Tacca M, Mey V, Funel N, Nannizzi S, Ricci S, Orlandini C, Boggi U, Campani D, Del Chiaro M, lannopollo M, Bevilacqua G, Mosca F, Danesi R: Transcription analysis of human equilibrative nucleoside transporter-1 predicts survival in pancreas cancer patients treated with gemcitabine. Cancer Res 2006, 66:3928-3935.

13. Marechal R, Mackey JR, Lai R, Demetter P, Peeters M, Polus M, Cass CE, Salmon I, Deviere J, Van Laethem JL: Deoxycitidine kinase is associated with prolonged survival after adjuvant gemcitabine for resected pancreatic adenocarcinoma. Cancer 2010, 116:5200-5206.

14. Nakahira S, Nakamori S, Tsujie M, Takahashi Y, Okami J, Yoshioka S, Yamasaki M, Marubashi S, Takemasa I, Miyamoto A, Takeda Y, Nagano H, Dono K, Umeshita K, Sakon M, Monden M: Involvement of ribonucleotide reductase $\mathrm{M} 1$ subunit overexpression in gemcitabine resistance of human pancreatic cancer. Int J Cancer 2007, 120:1355-1363.

15. Akita H, Zheng Z, Takeda Y, Kim C, Kittaka N, Kobayashi S, Marubashi S, Takemasa I, Nagano H, Dono K, Nakamori S, Monden M, Mori M, Doki Y, Bepler G: Significance of RRM1 and ERCC1 expression in resectable pancreatic adenocarcinoma. Oncogene 2009, 28:2903-2909.

16. Ohtaka K, Kohya N, Sato K, Kitajima Y, Ide T, Mitsuno M, Miyazaki K: Ribonucleotide reductase subunit $\mathrm{M} 1$ is a possible chemoresistance marker to gemcitabine in biliary tract carcinoma. Oncol Rep 2008, 20:279-286.

17. Marechal R, Bachet JB, Mackey JR, Dalban C, Demetter P, Graham K, Couvelard A, Surcek M, Bardier-Dupas A, Hammel P, Sauvanet A, Louvet C, Paye $F$, Rougier $P$, Penna $C$, André T, Dumontet $C$, Cass $C E$, Jordheim LP, Matera EL, Closset J, Salmon I, Devière J, Emile JF, Van Laethem JL: Levels of gemcitabine transport and metabolism proteins predict survival times of patients treated with gemcitabine for pancreatic adenocarcinoma. Gastroenterology 2012, 143:664-674. e1-e6.

18. Santini D, Schiavon G, Vincenzi B, Cass CE, Vasile E, Manazza AD, Catalano V, Baldi GG, Lai R, Rizzo S, Giacobino A, Chiusa L, Caraglia M, Russo A, Mackey J, Falcone A, Tonini G: Human equilibrative nucleoside transporter 1 ( $h E N T 1$ ) levels predict response to gemcitabine in patients with biliary tract cancer (BTC). Curr Cancer Drug Targets 2011, 11:123-129.

19. Borbath I, Verbrugghe L, Lai R, Gigot JF, Humblet Y, Piessevaux H, Sempoux C: Human equilibrative nucleoside transporter 1 (hENT1) expression is a potential predictive tool for response to gemcitabine in patients with advanced cholangiocarcinoma. Eur J Cancer 2012, 48:990-996.

20. Kobayashi H, Murakami Y, Uemura K, Sudo T, Hashimoto Y, Kondo N, Sueda T: Human equilibrative nucleoside transporter 1 expression predicts survival of advanced cholangiocarcinoma patients treated with gemcitabine-based adjuvant chemotherapy after surgical resection. Ann Surg 2012, 256:288-296.

21. Nakamura J, Kohya N, Kai K, Ohtaka K, Hashiguchi K, Hiraki M, Kitajima Y, Tokunaga O, Noshiro H, Miyazaki K: Ribonucleotide reductase subunit M1 assessed by quantitative double-fluorescence immunohistochemistry predicts the efficacy of gemcitabine in biliary tract carcinoma. Int J Oncol 2010, 37:845-852.

doi:10.1186/1477-7819-11-117

Cite this article as: Murata et al:: Prognostic predictive values of gemcitabine sensitivity-related gene products for unresectable or recurrent biliary tract cancer treated with gemcitabine alone. World Journal of Surgical Oncology 2013 11:117.

\section{Submit your next manuscript to BioMed Central and take full advantage of:}

- Convenient online submission

- Thorough peer review

- No space constraints or color figure charges

- Immediate publication on acceptance

- Inclusion in PubMed, CAS, Scopus and Google Scholar

- Research which is freely available for redistribution 